\title{
Expression and role of microRNA-1271 in the pathogenesis of osteosarcoma
}

\author{
GONGBIAO LU ${ }^{1 *}$, LIN DU $^{2 *}$, YI GUO $^{1}$, BAOHUA XING $^{1}$, JISHOU LU $^{1}$ and YANCHUN WEI ${ }^{1}$ \\ ${ }^{1}$ Department of Spine Surgery, Jining No. 1 People's Hospital, Jining, Shandong 272111; \\ ${ }^{2}$ College of Nursing, Jining Medical University, Jining, Shandong 272013, P.R. China
}

Received November 7, 2016; Accepted June 29, 2017

DOI: $10.3892 /$ etm.2017.5649

\begin{abstract}
The aim of the current study was to investigate the expression and role of microRNA (miR)-1271 in the pathogenesis of osteosarcoma, and the associated underlying mechanisms. Tissue samples from 45 patients with osteosarcoma were collected, while the 143B, MG-63 and U-2 OS osteosarcoma cell lines were also cultured. The expression levels of miR-1271 in the tissues and cells were detected with reverse transcription-quantitative polymerase chain reaction, and 143B osteosarcoma cells were subjected to miR-1271 manipulation. In addition, the cell proliferation, cell cycle progression, and migration and invasion abilities were assessed by Cell Counting Kit- 8 assay, flow cytometry and Transwell chamber assay, respectively. Tissue inhibitor of metalloproteinases 2 (TIMP2) expression level was also detected with western blot analysis. Dual-luciferase reporter assay was performed to investigate the interaction between miR-1271 and TIMP2. The results revealed that miR-1271 expression was significantly elevated in the osteosarcoma tissue and was closely correlated with the clinical TNM staging. The expression levels of miR-1271 were also upregulated in the osteosarcoma cells, with the highest expression observed in 143B cells. Inhibition of miR-1271 significantly inhibited the cell proliferation, G1/S phase transition, and the migration and invasion abilities of 143B cells, while it also resulted in upregulated TIMP2 expression in these cells. Furthermore, overexpression of TIMP2 significantly inhibited the cell proliferation, G1/S phase transition, and migration and invasion abilities of 143B cells. Dual-luciferase reporter assay demonstrated that miR-1271 targeted on the 3'-untranslated region of TIMP2 mRNA. In conclusion, the expression levels
\end{abstract}

Correspondence to: Dr Yi Guo, Department of Spine Surgery, Jining No. 1 People's Hospital, 6 Jiankang Road, Jining, Shandong 272111, P.R. China

E-mail: guoyi000@126.com

*Contributed equally

Key words: osteosarcoma, microRNA-1271, tissue inhibitor of metalloproteinases 2, 143B cell line of miR-1271 were significantly elevated in osteosarcoma tissues and cells. miR-1271 downregulated the expression of TIMP2 to promote the proliferation and enhance the migration and invasion abilities of 143B osteosarcoma cells, functioning as an oncogene.

\section{Introduction}

Osteosarcoma is the most common histological type of primary bone tumors, which are rare tumors in clinical practice $(1,2)$. Osteosarcoma usually occurs in the metaphyseal region that is rich in blood supply, and is characterized by early lung metastasis and high recurrence rate (3). The majority of patients with osteosarcoma are accompanied with micrometastasis at the initial diagnosis (4). At present, these patients are mainly subjected to the surgical treatment combined with radiotherapy and chemotherapy. However, the outcomes of surgical treatment are limited due to the high morbidity, while radiotherapy and chemotherapy may induce severe side effects and drug resistance, resulting in disease recurrence and poor prognosis. The 5-year survival rate for patients with osteosarcoma is $50-60 \%$, and the 5-year survival rate for patients with osteosarcoma accompanied with metastasis is only $20-30 \%(5,6)$. Therefore, it is of great importance to increase the early diagnostic rate for osteosarcoma to improve the disease prognosis. To date, the molecular mechanisms for the pathogenesis and metastasis of osteosarcoma remain unclear.

MicroRNA (miRNA or miR) is a class of small non-coding RNAs with a length of $18-22 \mathrm{nt}$, which are able to bind to the 3'-untranslated region (UTR) of target mRNAs and form a silencing complex, blocking the mRNA translation $(7,8)$. miRs are involved in almost all the pathophysiological processes in eukaryotic cells, and serve important roles in the regulation of tumorigenesis and distant metastasis (9). For instance, different miRs in the peripheral blood have been recognized as the diagnostic markers for colon cancer at different stages (10). miR-194 has been recognized as the early diagnostic marker for kidney cancer (11), while miR-373 promotes the invasion and metastasis of glioma cells via regulating the expression of CD44 and transforming growth factor $\beta$ receptor 2 (12). Furthermore, miRs have been demonstrated to serve important roles in the pathogenesis, proliferation, invasion and metastasis of osteosarcoma. For example, miR-497 inhibits the cell proliferation, invasion and metastasis of human osteosarcoma cells 
by targeting angiomotin (13). miR-133a blocks the invasion and metastasis of osteosarcoma by downregulating insulin-like growth factor 1 receptor (14). In addition, miR-21 promotes the pathogenesis and development of osteosarcoma via the phosphatase and tensin homologue/protein kinase B pathway (15). miR-1271 is a recently discovered miR molecule (16) that has been reported to function as an oncogene or tumor-suppressor genes in several tumors (17). However, its expression and biological function in osteosarcoma pathogenesis have not yet been fully elucidated.

In the present study, the expression and role of miR-1271 in the pathogenesis of osteosarcoma were investigated in human tissues and three cell lines. Osteosarcoma cells were subjected to transfection with miR-1271 overexpressing plasmid. The cell proliferation, cell cycle progression, and migration and invasion abilities were evaluated. Previously, it has been demonstrated that the tissue inhibitor of metalloproteinases 2 (TIMP2) is closely associated with the proliferation, and invasion and metastasis of tumor cells. The downregulated TIMP2 expression is able to activate the matrix metalloproteinase (MMP) family proteins, further promoting the metastasis of tumor cells (18). Based on these findings, the expression of TIMP2 was also detected in the current study, and the effects of miR-1271 on TIMP2 expression were investigated. Furthermore, the dual-luciferase reporter assay was performed to confirm the interaction between miR-1271 and TIMP2.

\section{Materials and methods}

Study subjects and sample collection. In total, 45 patients with osteosarcoma (31 males and 14 females; mean age, $22.6 \pm 1.6$ years) were included into the present study. These patients received curative or palliative resection at Jining No. 1 People's Hospital (Jining, China) between October 2013 and November 2015. According to TNM staging (19), these patients were diagnosed and classified by two pathologists into 19 cases of T1 stage, 18 cases of T2 stage and 8 cases of T3 stage. Tumor samples were collected, and the adjacent normal tissues ( $5 \mathrm{~cm}$ away from the tumor tissue) were harvested as the control tissues. The tissues were flash frozen in liquid nitrogen and ground into powder. Prior written informed consent was obtained from every patient, and the study was approved by the Ethics Review Board of the Jining No. 1 People's Hospital.

Cell lines and cell culture. 143B (cat. no. CRL-8303), MG-63 (cat. no. CRL-1427) and U-2 OS (cat. no. HTB-96) osteosarcoma cell lines were purchased from American Type Culture Collection (ATCC; Rockville, MD, USA). Cells separated from tumor-adjacent tissues were used as control for the three types of osteosarcoma cells. These cells were cultured with the RPMI-1640 complete medium (Gibco; Thermo Fisher Scientific, Inc., Waltham, MA, USA) in a $37^{\circ} \mathrm{C}$ and $5 \% \mathrm{CO}_{2}$ incubator. The culture medium was changed every other day.

Reverse transcription-quantitative polymerase chain reaction (RT-qPCR). Total RNA was extracted with the TRIzol reagent (Thermo Fisher Scientific, Inc.) from the tissue and cell samples. Using NanoDrop 2000 (Thermo Fisher Scientific, Inc.), RNA quality was measured using OD260/280 values and the concentration of RNA was obtained. Using a miRcute
miRNA cDNA First Chain Synthesis Kit (cat. no. KR201; Tiangen, Beijing, China), cDNA was then obtained with polyA tailing method (20). The RT reaction was performed at $37^{\circ} \mathrm{C}$ for $60 \mathrm{~min}$. qPCR was performed on the Step One plus Real-Time PCR System (Applied Biosystems; Thermo Fisher Scientific, Inc.). The reaction system $(20 \mu \mathrm{l})$ contained $10 \mu \mathrm{l}$ qRT-PCR-Mix, $0.5 \mu \mathrm{l}$ upstream primer (5'-CTTGGC ACCTAGCAAGCACTCA-3'), $0.5 \mu \mathrm{l}$ downstream universal primer (5'-TGGTGTCGTGGAGTCG-3'), $2 \mu \mathrm{l}$ cDNA and $7 \mu 1$ $\mathrm{ddH}_{2} \mathrm{O}$. The reaction protocol was: initial denaturation at $95^{\circ} \mathrm{C}$ for $10 \mathrm{~min}$; 40 cycles of $95^{\circ} \mathrm{C}$ for $1 \mathrm{~min}$ and $60^{\circ} \mathrm{C}$ for $30 \mathrm{sec}$. To measure TIMP2 mRNA expression, an miRcute enhanced miRNA fluorescence quantitative detection kit (FP411, Tiangen) was used. The primers for TIMP2 were 5'-GAGCGA GAAGGAGGTGGATTCCGGG-3' (forward) and 5'-ATG TCAAGA AACTCCTGCTTCGGGGG-3' (reverse)/The internal control was U6 (forward, 5'-CTCGCTTCGGCAGCA CA-3'; reverse, 5'-AACGCTTCACGAATTTGCGT-3'). The expression of miR-1271 was assessed using a BeyoFast SYBR Green qPCR Mix (Beyotime Institute of Biotechnology. Haimen, China). The forward primer for miR-1271 was 5'-CTTGGCACCTAGCAAGCACTCA-3', and the reverse primer for miR-1271 was a universal primer provided in the kit. The internal control for miR-1271 was GAPDH (forward, 5'-CGGAGTCAACGGATTTGGTCGTAT-3'; reverse, 5'-AGC CTTCTCCATGGTGGTGAAGAC-3'). The $2^{-\Delta \Delta C q}$ method was used to calculate the relative expression of TIMP 2 mRNA or miR-1271 against internal references (21).

Cell transfection. Osteosarcoma 143B cells were seeded into a 24-well plate at a density of $1 \times 10^{5}$ cells/well, and cultured with the RPMI-1640 medium containing $10 \%$ fetal bovine serum (FBS) at $37^{\circ} \mathrm{C}$ in an atmosphere containing $5 \% \mathrm{CO}_{2}$ overnight. Next, $50 \mu \mathrm{l}$ Opti Memi culture medium and $2 \mu \mathrm{l}$ Lipofectamine 2000 (both Thermo Fisher Scientific, Inc.) was added to the first Eppendorf (EP) tube. A second EP tube was filled with $50 \mu \mathrm{l}$ Opti Memi culture medium, along with $1.25 \mu \mathrm{l}$ miR-1271 inhibitor (20 $\mu \mathrm{M}$; RiboBio Co., Ltd., Guangzhou, China), negative control (NC) or pcDNA3.1-TIMP2 plasmid (RiboBio Co., Ltd.). Following $2 \mathrm{~min}$ of incubation, the contents of the second EP tube (miR-1271 inhibitor, NC or pcDNA3.1-TIMP2) and the first EP tubes were mixed together, forming the different study groups. After $15 \mathrm{~min}$, the various mixtures were transferred into the wells and incubated for 6 h. Subsequently, the cells were cultured with RPMI 1640 medium containing $10 \%$ FBS for $48 \mathrm{~h}$.

Cell Counting Kit (CCK)-8 assay. The cell proliferation was assessed with the CCK- 8 assay. Following the various treatments, the $143 \mathrm{~B}$ cells were planted onto a 96 -well plate at a density of 5,000 cells/well. Every $24 \mathrm{~h}$, the cells were treated with the CCK- 8 reaction solution (Beyotime Institute of Biotechnology) for $30 \mathrm{~min}$, for 3 consecutive days. The absorbance at $490 \mathrm{~nm}$ was then read with a microplate reader, and the cell proliferation curve was obtained accordingly.

Flow cytometry for determination of cell cycle progression. Cell cycle was detected by flow cytometry. At $24 \mathrm{~h}$ after cell transfection, $1 \times 10^{6}$ cells were washed with phosphate-buffered saline. The cell cycle was then examined with the Cell Cycle 
Assay kit (BD Biosciences, Franklin Lakes, NJ, USA), according to the manufacturer's instructions. Briefly, the cells were incubated with $200 \mu \mathrm{l}$ solution $\mathrm{A}$ at room temperature for $10 \mathrm{~min}$, followed by incubation with $150 \mu \mathrm{l}$ solution B at room temperature for $10 \mathrm{~min}$, and subsequent incubation with $120 \mu 1$ solution $\mathrm{C}$ in the dark for $10 \mathrm{~min}$. Fluorescence was detected with a flow cytometer (BD Biosciences), and the results were analyzed with the Modifit software (version 3.1; Verity Software House, Topsham, ME, USA).

Transwell chamber detection. The migration and invasion abilities of 143B cells were assessed using a 24-well plate with Transwell chambers (pore size, $8 \mu \mathrm{m}$; Corning Inc., Corning, NY, USA). A total of $2 \times 10^{5}$ cells in $200 \mu \mathrm{l}$ serum-free RPMI 1640 medium were planted onto the upper chamber, and $500 \mu \mathrm{l}$ RPMI-1640 medium containing 10\% FBS was added to the lower chamber. After $24 \mathrm{~h}$, the cells in the upper chamber were removed, followed by fixation of all cells with $4 \%$ formaldehyde at room temperature for $10 \mathrm{~min}$ and subsequent Giemsa staining. Under a light microscope (magnification, x200), the number of membrane-penetrating cells were counted in five randomly selected fields, and the cell migration ability was assessed accordingly.

To evaluate the cell invasion ability, a Matrigel matrix (BD Biosciences) was used to simulate the extracellular environment. Subsequent to diluting with serum-free RPMI-1640 medium (1:2 dilution), the matrix (100 $\mu \mathrm{l})$ was evenly smeared onto the upper chamber and incubated at $37^{\circ} \mathrm{C}$ for $60 \mathrm{~min}$ to obtain the gel. The experimental procedures for cell invasion investigation were the same as for the migration ability assessment, but were performed on ice. The results were observed under a light microscope (magnification, x200) $72 \mathrm{~h}$ later.

Western blot analysis. Following transfection, the cells were lysed with the radioimmunoprecipitation assay lysis buffer containing phenylmethane sulfonyl fluoride (Beyotime Institute of Biotechnology). Subsequent to centrifugation at $12,000 \times \mathrm{g}$ and $4^{\circ} \mathrm{C}$ for $10 \mathrm{~min}$, the protein concentration was measured with the BCA method. A protein sample $(5 \mu \mathrm{l})$ was then subjected to $12 \%$ SDS-PAGE and electronically transferred onto a polyvinylidene difluoride membrane. After blocking with non-fat milk at room temperature for $1 \mathrm{~h}$, the membrane was incubated with rabbit anti-human anti-TIMP2 antibody (1:1,000 dilution; cat. no. ab180630; Abcam, Cambridge, UK) or mouse anti-human anti-GAPDH antibody (1:5,000 dilution; cat. no. ab8245; Abcam) at $4^{\circ} \mathrm{C}$ overnight. Subsequently, the membrane was incubated with a horseradish peroxidase-conjugated goat anti-mouse (1:5,000 dilution; cat. no. ab6789; Abcam) or goat anti-rabbit (1:2,000 dilution; cat. no. ab6721; Abcam) secondary antibodies, respectively, at room temperature for $1 \mathrm{~h}$. Color development was performed with the enhanced chemiluminescence kit (Beyotime Institute of Biotechnology), and the protein bands were then imaged and analyzed using Quantity One software (version 4.62; Bio-Rad, Hercules, CA, USA).

Bioinformatics prediction and dual-luciferase reporter assay. The target gene of miR-1271 was predicted with bioinformatics analysis using the TargetScan online tool (www.targetscan.org). Based on the findings that TIMP2 was a target gene of miR-1271, the wild-type (5'-UAAACU GACGAUAUACAGGCACA-3') and mutant (5'-UAAACU GACGAUAUAGAAGAAGA-3') seed regions of the 3'-UTR of TIMP2 gene were synthesized with the Spe-1 and HindIII restriction sites at the ends. Next, these regions were cloned into the pMIR-REPORT luciferase reporter plasmid (Thermo Fisher Scientific, Inc.). The miR-1271 mimics (HanBio Biotechnology, Shanghai, China), along with $0.5 \mu \mathrm{g}$ plasmid containing wild-type or mutant 3'-UTR of TIMP2 mRNA, were co-transfected into the HEK293T cells (Cell Bank, Chinese Academy of Sciences, Shanghai, China) at $37^{\circ} \mathrm{C}$ using Lipofectamine $^{\circledR} 2000$ (Thermo Fisher Scientific, Inc.). After $24 \mathrm{~h}$, the cells were lysed with the dual-luciferase reporter kit (Promega Corp., Madison, WI, USA), and the fluorescence was detected with the GloMax 20/20 luminometer (Promega Corp.). Renilla was used as the internal reference.

Statistical analysis. Data are expressed as the mean \pm standard deviation. SPSS version 17.0 software (SPSS, Inc., Chicago, IL, USA) was used for statistical analysis. The Student's t-test was performed for group comparison, followed by Scheffe test as post hoc test. $\mathrm{P}<0.05$ was considered to indicate a statistically significant difference.

\section{Results}

Expression of miR-1271 in osteosarcoma tissues and cells. To investigate the expression of miR-1271 in the osteosarcoma tissues and cell lines, RT-qPCR analysis was performed. The results revealed that, compared with the adjacent normal tissue, the miR-1271 expression level was significantly elevated in the osteosarcoma tissue $(\mathrm{P}<0.05$; Fig. $1 \mathrm{~A})$. When considering the TNM staging, the miR-1271 expression in the T3 group was significantly higher compared with that in the T1 and T2 groups (both $\mathrm{P}<0.05$; Fig. 1B). Furthermore, the expression of miR-1271 was significantly elevated in the three osteosarcoma cell lines compared cells from tumor-adjacent tissues, with the highest expression observed in the 143B cells $(\mathrm{P}<0.05$; Fig. 1C). These results suggest that the expression of miR-1271 is significantly elevated in the osteosarcoma tissues and cells, which may be closely associated with the disease pathogenesis.

Effects of miR-1271 on the proliferation, cell cycle progression, and migration and invasion abilities of $143 \mathrm{~B}$ osteosarcoma cells. The effects of miR-1271 on the proliferation, cell cycle progression, and migration and invasion abilities of 143B osteosarcoma cells were investigated. The cells were first transfected with an miR-1271 inhibitor, and the results indicated that the level of miR-1271 in cells transfected with miR-1271 inhibitor was significantly lower than compared with cells transfected with miR-NC (data not shown). The results from the CCK-8 assay demonstrated that the inhibition of miR-1271 significantly decreased the proliferation of 143B osteosarcoma cells ( $\mathrm{P}<0.05$; Fig. $2 \mathrm{~A})$, indicating that miR-1271 may promote the proliferation of osteosarcoma cells. In addition, the results of flow cytometry indicated that, compared with the NC group, 143B osteosarcoma cells transfected with miR-1271 inhibitor were arrested at the G1/S phase (Fig. 2B). This suggests that upregulated expression of miR-1271 may improve the G1/S 

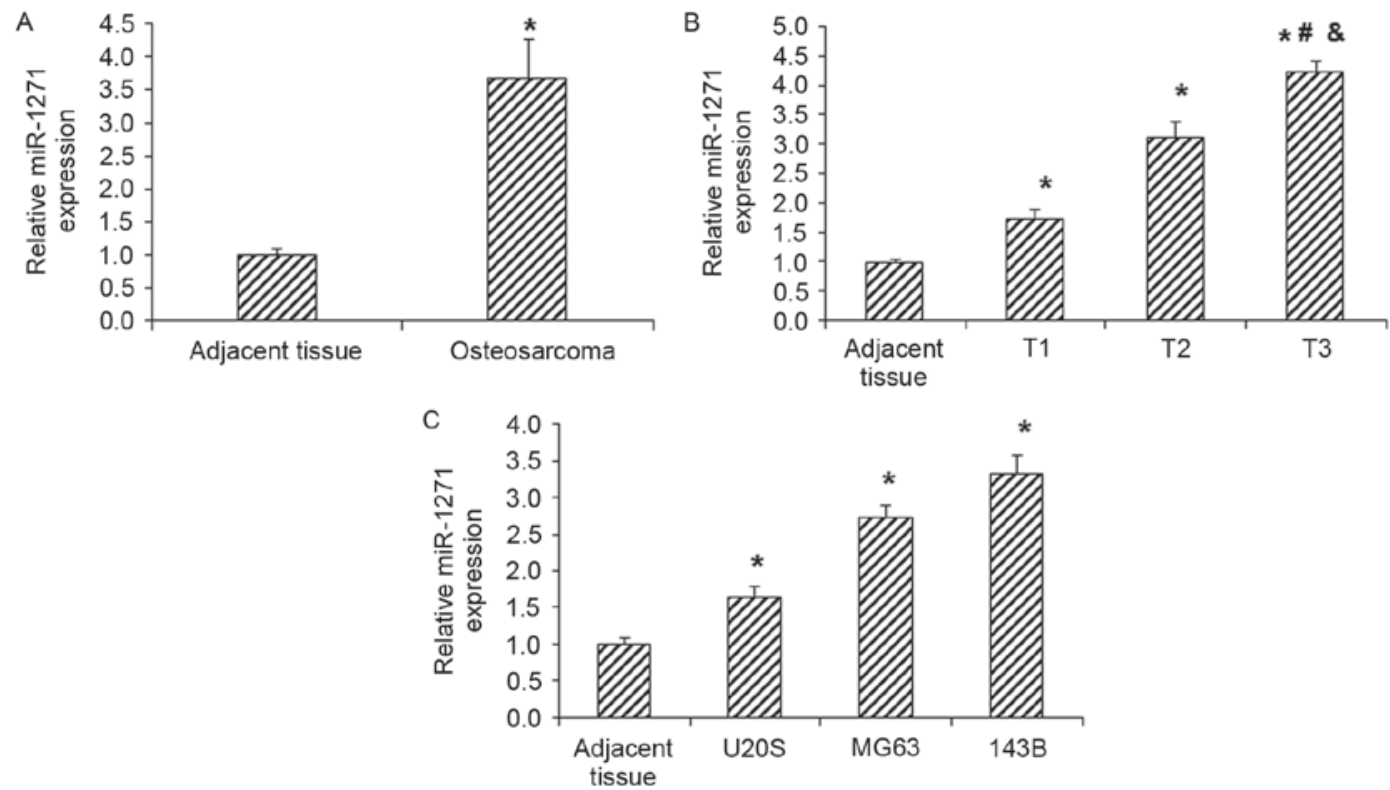

Figure 1. Expression levels of miR-1271 in the osteosarcoma tissues and cell lines. Reverse transcription-quantitative polymerase chain reaction was performed to detect the expression levels of miR-1271 in the: (A) Osteosarcoma and adjacent normal tissues; (B) normal tissue and osteosarcoma tissues at different TNM stages (T1, T2 and T3 stages); and (C) osteosarcoma cell lines U-2 OS, MG-63 and 143B. n=3. ${ }^{~} \mathrm{P}<0.05$ vs. adjacent normal tissue; ${ }^{\sharp} \mathrm{P}<0.05$ vs. T1 group; \& $\mathrm{P}<0.05$ vs. T2 group. miR, microRNA.
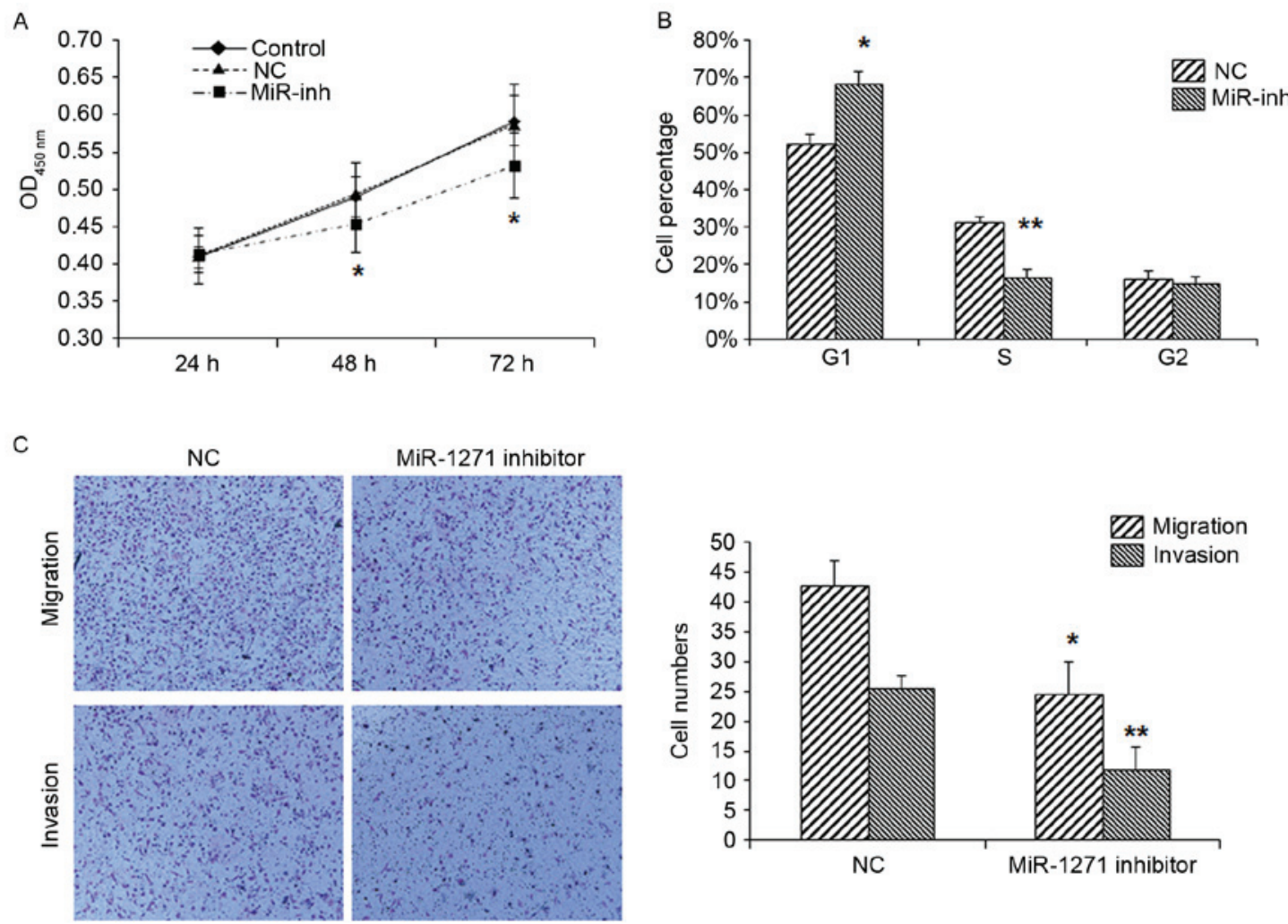

Figure 2. Effects of miR-1271 on the (A) proliferation, (B) cell cycle progression, and (C) migration and invasion abilities of 143B osteosarcoma cells. Following transfection with MiR-inh or $\mathrm{NC}$ for $6 \mathrm{~h}$, the proliferation, cell cycle progression, and migration and invasion abilities were evaluated by the Cell Counting Kit-8 assay, flow cytometry and Transwell chamber assay, respectively. $\mathrm{n}=3$. ${ }^{*} \mathrm{P}<0.05$ and ${ }^{* *} \mathrm{P}<0.01$, vs. NC group. MiR-inh, miR-1271 inhibitor; NC, negative control; OD, optical density; miR, microRNA.

phase transition and subsequently promote the proliferation of 143B osteosarcoma cells. Furthermore, the Transwell chamber migration assay demonstrated that, compared with the $\mathrm{NC}$ group, the penetrating cells were significantly less in the 143B osteosarcoma cells transfected with the miR-1271 inhibitor $(\mathrm{P}<0.05$; Fig. $2 \mathrm{C})$, indicating declined migration ability of these transfected cells. The invasion assay revealed that the penetrating 143B cells were significantly reduced compared 
A

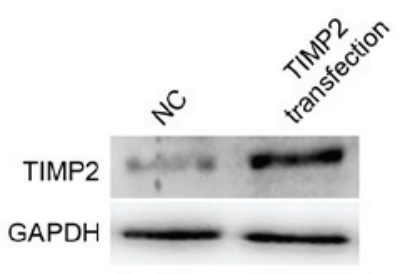

B

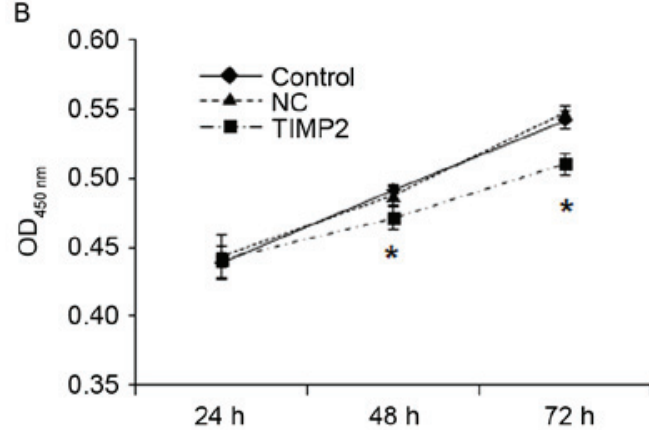

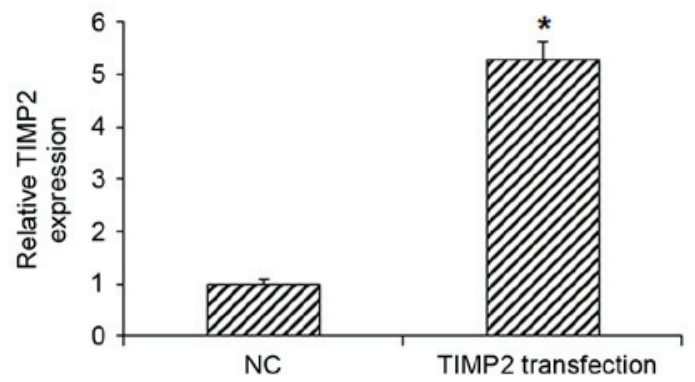

C

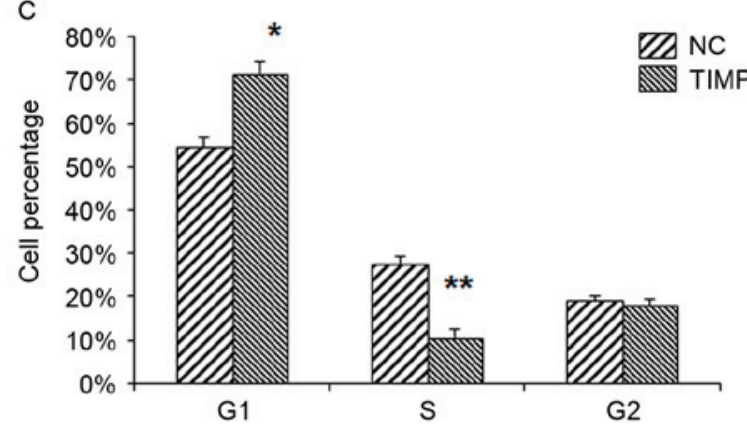

D
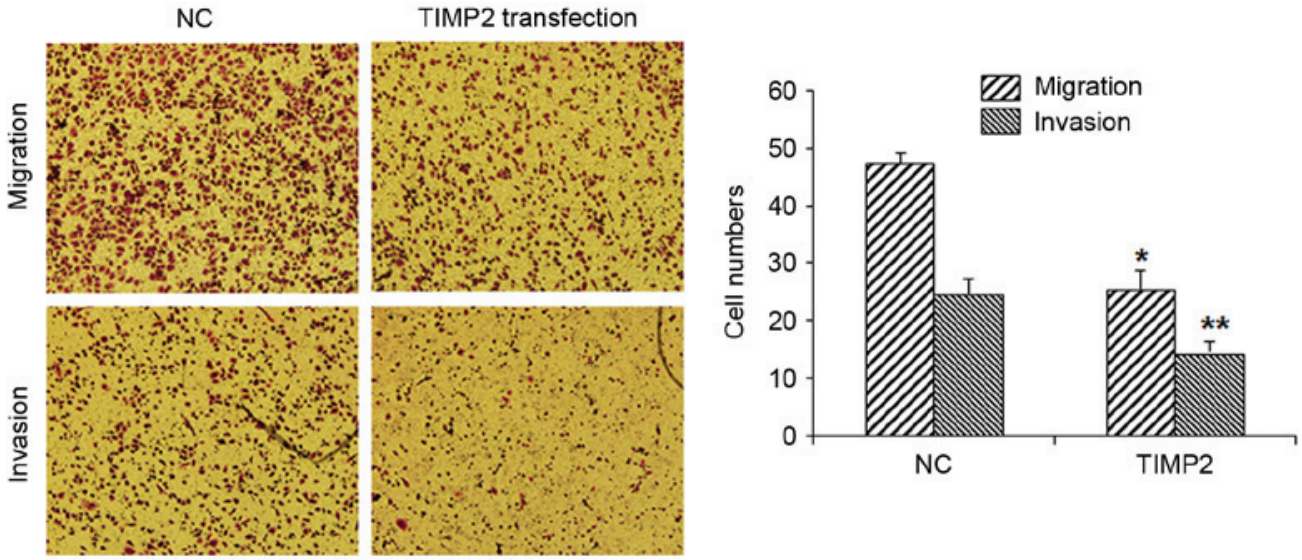

Figure 3. Effects of TIMP2 overexpression on the proliferation, cell cycle progression, and migration and invasion abilities of 143B osteosarcoma cells. (A) Overexpression of TIMP2 in the 143B osteosarcoma cells was detected with western blot analysis. Following transfection with TIMP2 for $6 \mathrm{~h}$, the (B) proliferation, (C) cell cycle progression, and (D) migration and invasion abilities of 143B osteosarcoma cells were evaluated with the Cell Counting Kit-8 assay, flow cytometry and Transwell chamber assay, respectively. $n=3$. ${ }^{*} \mathrm{P}<0.05$ and ${ }^{* *} \mathrm{P}<0.01$ vs. NC group. NC, negative control; OD, optical density; TIMP2, tissue inhibitor of metalloproteinases 2; miR, microRNA.

with those in the $\mathrm{NC}$ group $(\mathrm{P}<0.05$; Fig. $2 \mathrm{C})$, indicating that miR-1271 may inhibit the invasion ability of osteosarcoma cells. Taken together, these results suggest that miR-1271 may significantly increase the proliferation, enhance the migration and invasion abilities, and promote the G1/S phase transition of osteosarcoma cells.

Biological functions of TIMP2 and its interaction with miR-1271 in 143B osteosarcoma cells. The biological functions of TIMP2 and its interaction with miR-1271 in 143B osteosarcoma cells were next investigated. Overexpression of TIMP2 was initially induced in the osteosarcoma cells by plasmid transfection (Fig. 3A), and then the proliferation, cell cycle progression, and migration and invasion abilities of cells were evaluated with the CCK- 8 assay, flow cytometry and Transwell chamber assay, respectively. The results identified that TIMP2 overexpression significantly decreased the cell proliferation, blocked the G1/S phase transition, and decreased the migration and invasion abilities of osteosarcoma cells (Fig. 3B-D). These results suggest that TIMP2 may serve as a tumor-suppressing gene, while its overexpression may inhibit the pathogenesis and development of osteosarcoma.

Western blot analysis demonstrated that transfection with the miR-1271 inhibitor significantly elevated the expression of TIMP2 in the osteosarcoma cells $(\mathrm{P}<0.05$; Fig. 4A). The results from the dual-luciferase reporter assay revealed that, compared with the $\mathrm{NC}$ group, the fluorescence density was significantly reduced in the HEK293T cells co-transfected with miR-1271 mimics and pMIR-REPORT-wild-type luciferase reporter $(\mathrm{P}<0.05)$. By contrast, no significant differences in the fluorescence density were observed when these cells were co-transfected with miR-1271 mimics and pMIR-REPORT-mutant luciferase reporter $(\mathrm{P}>0.05$; 
A
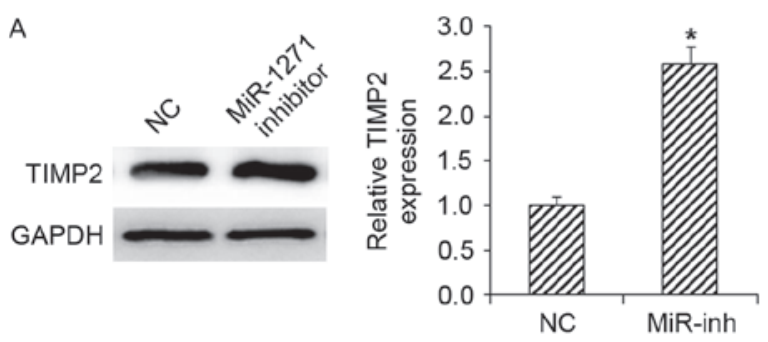

B

\section{5'... UAAACUGACGAUAUACAGGCACA... \\ |||||| $\mid$ \\ 3' ACggaccguguaucguccguga}

C

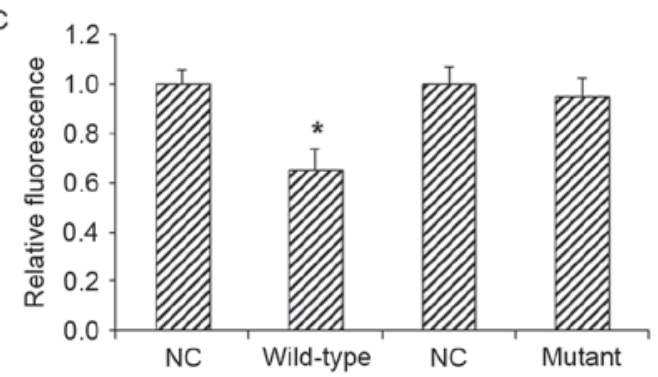

Figure 4. Interaction between miR-1271 and TIMP2. (A) After transfected with MiR-inh or NC for $6 \mathrm{~h}$, the expression levels of TIMP2 in the 143B osteosarcoma cells were assessed by western blot analysis. (B) The predicted target gene of miR-1271 by bioinformatics analysis using the TargetScan tool. (C) Dual-luciferase reporter assay, in which HEK293T cells were co-transfected with the miR-1271 mimics, along with pMIR-REPORT-wild-type or pMIR-REPORT-mutant luciferase reporter. After $24 \mathrm{~h}$, the fluorescence of cell lysate was detected. $\mathrm{n}=3$. ${ }^{*} \mathrm{P}<0.05$ vs. NC group. TIMP2, tissue inhibitor of metalloproteinases 2; NC, negative control; MiR-inh, miR-1271 inhibitor; miR, microRNA.

Fig. 4B and C). These results suggest that miR-1271 may bind to the 3'-UTR of TIMP2 mRNA.

\section{Discussion}

In the present study, the results of RT-qPCR demonstrated that the expression of miR-1271 was significantly elevated in the osteosarcoma tissue, and this expression was negatively correlated with the clinical TNM staging of patients. However, downregulation of miR-1271 significantly inhibited the proliferation, migration and invasion of 143B osteosarcoma cells. Next, bioinformatics prediction revealed that TIMP2 may be the target gene for miR-1271, while transfection with plasmids to induce overexpression of TIMP2 significantly inhibited the proliferation, as well as the migration and invasion abilities, of 143B osteosarcoma cells. Dual-luciferase reporter assay also displayed that miR-1271 was able to directly bind to the 3'-UTR of TIMP2 mRNA and regulate the gene expression. According to these results, it can be suggested that miR-1271 functions as oncogene in the pathogenesis of osteosarcoma, while miR-1271 may downregulate the expression of TIMP2 and promote the pathogenesis and development of osteosarcoma.

miR-1271 is a recently identified miR molecule with a relatively limited number of investigations into its role and function in tumor pathogenesis. Increasing studies have suggested that miR-1271 functions as an oncogene in tumorigenesis. Zhou et al (22) observed that miR-1271 is downregulated in non-small cell lung cancer, which may inhibit the tumor cell proliferation. Liu et al (23) have indicated that miR-1271 targets cyclinG1 to inhibit the growth of ovarian cancer. The results of the present study demonstrated that the expression of miR-1271 was upregulated in the osteosarcoma tissue, while it was negatively correlated with the TNM staging. In the osteosarcoma cells, the highest expression of miR-1271 was observed in the 143B cells. The in vitro results revealed that miR-1271 significantly promoted the proliferation of 143B osteosarcoma cells. It has also been reported that miR-1271 was able to inhibit the invasion and metastasis of various tumors. For instance, in gastric cancer, miR-1271 targeted forkhead box Q1 expression to inhibit the epithelial-mesenchymal transition and metastasis of gastric cancer cells (24). In squamous cell carcinoma, miR-1271 downregulated the expression of alkaline phosphatase to block the invasion and metastasis of tumor cells (25). The current study results demonstrated that the inhibited expression of miR-1271 significantly reduced the migration and invasion abilities of 143B osteosarcoma cells, indicating that miR-1271 was able to promote the pathogenesis of osteosarcoma.

Bioinformatics prediction in the present study indicated that TIMP2 was one of the target genes for miR-1271. TIMP2 is a main member of the MMP inhibitors, which is able to inhibit the activity of MMP-2, suppress the degradation of the extracellular matrix, and thereby block the invasion and metastasis of tumors (26). A previous study demonstrated that the expression of TIMP2 is associated with the invasion and metastasis of various tumors, which may be used as a treatment target (27). However, the regulating mechanisms of TIMP2 remain unclear (28). The current study results revealed that upregulation of TIMP2 expression reduced the proliferation of 143B osteosarcoma cells, inhibited the cell G1/S phase transition, and attenuate the migration and invasion abilities of cells. The dual-luciferase reporter assay also revealed that miR-1271 directly targeted the 3'-UTR of TIMP2 mRNA and regulated the gene expression of TIMP2, thereby functioning as an oncogene.

In conclusion, the results of the present study revealed that the miR-1271 expression levels were significantly elevated in the osteosarcoma tissues and cells. In addition, inhibition of miR-1271 significantly inhibited the cell proliferation, G1/S phase transition, and the migration and invasion abilities of 143B osteosarcoma cells, and resulted in upregulated TIMP2 expression. Furthermore, overexpression of TIMP2 significantly inhibited the cell proliferation, G1/S phase transition, and migration and invasion metastasis abilities of 143B osteosarcoma cells. Dual-luciferase reporter assay also indicated a direct interaction between miR-1271 and TIMP2. These results suggest that miR-1271 may downregulate the expression of TIMP2 to promote the pathogenesis and development of osteosarcoma, functioning as an oncogene; thus, miR-1271 may be a potential treatment target for osteosarcoma in clinical practice.

\section{Acknowledgements}

The authors would like to thank Dr Liuzhong Jin (Department of Spine Surgery, Jining No. 1 People's Hospital, Jining, China) for his valuable help. 


\section{References}

1. McDougall JA, Bansal A, Goulart BH, McCune JS, Karnopp A, Fedorenko C, Greenlee S, Valderrama A, Sullivan SD and Ramsey SD: The clinical and economic impacts of skeletal-related events among medicare enrollees with prostate cancer metastatic to bone. Oncologist 21: 320-326, 2016.

2. Yamagishi T, Kawashima H, Ogose A, Sasaki T, Hotta T, Inagawa $\mathrm{S}$, Umezu $\mathrm{H}$ and Endo N: Disappearance of giant cells and presence of newly formed bone in the pulmonary metastasis of a sacral giant-cell tumor following denosumab treatment: A case report. Oncol Lett 11: 243-246, 2016.

3. He J, Zhang P, Li Q, Zhou D and Liu P: Expression of high mobility group box 1 protein predicts a poorer prognosis for patients with osteosarcoma. Oncol Lett 11: 293-298, 2016.

4. Cheng HL, Lin CW, Yang JS, Hsieh MJ, Yang SF and Lu KH: Zoledronate blocks geranylgeranylation not farnesylation to suppress human osteosarcoma U2OS cells metastasis by EMT via Rho A activation and FAK-inhibited JNK and p38 pathways. Oncotarget 7: 9742-9758, 2016.

5. Stine KC, Wahl EC, Liu L, Skinner RA, VanderSchilden J, Bunn RC, Montgomery CO, Aronson J, Becton DL, Nicholas RW, et al: Nutlin-3 treatment spares cisplatin-induced inhibition of bone healing while maintaining osteosarcoma toxicity. J Orthop Res 34: 1716-1724, 2016.

6. Xie GP, Song HJ, Jiang N, Qin CH, Wang L, Xu SY and Yu B: Periosteal osteosarcoma and Marfan's syndrome: A case report and literature review. Oncol Lett 11: 311-315, 2016.

7. Fanini $\mathrm{F}$ and Fabbri M: microRNAs and cancer resistance: A new molecular plot. Clin Pharmacol Ther 99: 485-493, 2016.

8. Zhu L, Zhao J, Wang J, Hu C, Peng J, Luo R, Zhou C, Liu J, Lin J, Jin Y, et al: MicroRNAs are involved in the regulation of ovary development in the pathogenic blood fluke schistosoma japonicum. PLoS Pathog 12: e1005423, 2016

9. Wang Y, Chen J, Lin Z, Cao J, Huang H, Jiang Y, He H, Yang L, Ren N and Liu G: Role of deregulated microRNAs in non-small cell lung cancer progression using fresh-frozen and formalin-fixed, paraffin-embedded samples. Oncol Lett 11: 801-808, 2016

10. Sun Y, Liu Y, Cogdell D, Calin GA, Sun B, Kopetz S, Hamilton SR and Zhang W: Examining plasma microRNA markers for colorectal cancer at different stages. Oncotarget 7: 11434-11449, 2016.

11. Nofech-Mozes R, Khella HW, Scorilas A, Youssef L, Krylov SN, Lianidou E, Sidiropoulos KG, Gabril M, Evans A and Yousef GM: MicroRNA-194 is a marker for good prognosis in clear cell renal cell carcinoma. Cancer Med 5: 656-664, 2016.

12. Wei F, Wang Q, Su Q, Huang H, Luan J, Xu X and Wang J: miR-373 Inhibits glioma cell U251 migration and invasion by down-regulating CD44 and TGFBR2. Cell Mol Neurobiol 36: 1389-1397, 2016.

13. Ruan WD, Wang P, Feng S, Xue Y and Zhang B: MicroRNA-497 inhibits cell proliferation, migration, and invasion by targeting AMOT in human osteosarcoma cells. Onco Targets Ther 9: 303-313, 2016.

14. Chen G, Fang T, Huang Z, Qi Y, Du S, Di T, Lei Z, Zhang X and Yan W: MicroRNA-133a inhibits osteosarcoma cells proliferation and invasion via targeting IGF-1R. Cell Physiol Biochem 38: 598-608, 2016.
15. Lv C, Hao Y and Tu G: MicroRNA-21 promotes proliferation, invasion and suppresses apoptosis in human osteosarcoma line MG63 through PTEN/Akt pathway. Tumour Biol 37: 9333-9342, 2016.

16. Liang HF, Zhang XZ, Liu BG, Jia GT and Li WL: Circular RNA circ-ABCB10 promotes breast cancer proliferation and progression through sponging miR-1271. Am J Cancer Res 7: 1566-1576, 2017.

17. Liu H, Wang H, Liu X and Yu T: miR-1271 inhibits migration, invasion and epithelial-mesenchymal transition by targeting ZEB1 and TWIST1 in pancreatic cancer cells. Biochem Biophys Res Commun 472: 346-352, 2016.

18. Jia J, Li F, Tang XS, Xu S, Gao Y, Shi Q, Guo W, Wang X, He D and Guo P: Long noncoding RNA DANCR promotes invasion of prostate cancer through epigenetically silencing expression of TIMP2/3. Oncotarget 7: 37868-37881, 2016.

19. Baumhoer D: Pathogenesis and genetics of osteosarcoma: Current concepts and developments. Pathologe, Sep 19, 2017 (In German).

20. Ozsolak F, Goren A, Gymrek M, Guttman M, Regev A, Bernstein BE and Milos PM: Digital transcriptome profiling from attomole-level RNA samples. Genome Res 20: 519-525, 2010.

21. Livak KJ and Schmittgen TD: Analysis of relative gene expression data using real-time quantitative PCR and the 2(-Delta Delta C(T)) method. Methods 25: 402-408, 2001.

22. Zhou Z, Niu X, Li C, Sheng S and Lu S: Inhibition of the growth of non-small cell lung cancer by miRNA-1271. Am J Transl Res 7: 1917-1924, 2015.

23. Liu X, Ma L, Rao Q, Mao Y, Xin Y, Xu H, Li C and Wang X: MiR-1271 inhibits ovarian cancer growth by targeting cyclin G1. Med Sci Monit 21: 3152-3158, 2015.

24. Xiang XJ, Deng J, Liu YW, Wan LY, Feng M, Chen J and Xiong JP: MiR-1271 inhibits cell proliferation, invasion and EMT in gastric cancer by targeting FOXQ1. Cell Physiol Biochem 36: 1382-1394, 2015

25. Kong D, Zhang G, Ma H and Jiang G: miR-1271 inhibits OSCC cell growth and metastasis by targeting ALK. Neoplasma 62: 559-566, 2015.

26. Yan A, Yang C, Chen Z, Li C and Cai L: MiR-761 promotes progression and metastasis of non-small cell lung cancer by targeting ING4 and TIMP2. Cell Physiol Biochem 37: 55-66, 2015.

27. Yi X, Guo J, Guo J, Sun S, Yang P, Wang J, Li Y, Xie L, Cai J and Wang Z: EZH2-mediated epigenetic silencing of TIMP2 promotes ovarian cancer migration and invasion. Sci Rep 7: $3568,2017$.

28. Lu GJ, Dong YQ, Zhang QM, Di WY, Jiao LY, Gao QZ and Zhang CG: miRNA-221 promotes proliferation, migration and invasion by targeting TIMP2 in renal cell carcinoma. Int J Clin Exp Pathol 8: 5224-5229, 2015. International (CC BY-NC-ND 4.0) License. 